Research Paper

\title{
Expression of epicardial adipose tissue thermogenic genes in patients with reduced and preserved ejection fraction heart failure
}

Luis M. Pérez-Belmonte ${ }^{1 \bowtie *}$, Inmaculada Moreno-Santos ${ }^{1 *}$, Juan J. Gómez-Doblas ${ }^{1}$, José M. García-Pinilla ${ }^{1}$, Luis Morcillo-Hidalgo ${ }^{1}$, Lourdes Garrido-Sánchez², Concepción Santiago-Fernández², María G. Crespo-Leiro ${ }^{3}$, Fernando Carrasco-Chinchillaㄹ, Pedro L. Sánchez-Fernández ${ }^{4}$, Eduardo de Teresa-Galván", Manuel Jiménez-Navarro ${ }^{1 凶}$

1. Unidad de Gestión Clínica Área del Corazón, Instituto de Investigación Biomédica de Málaga (IBIMA), Hospital Universitario Virgen de la Victoria, Universidad de Málaga (UMA), CIBERCV Enfermedades Cardiovasculares, Málaga, Spain.

2. Unidad de Gestión Clínica de Endocrinología y Nutrición, Instituto de Investigación Biomédica de Málaga (IBIMA), Hospital Universitario Virgen de la Victoria, Málaga, CIBER Fisiopatología Obesidad y Nutrición (CIBERobn), Instituto de Salud Carlos III, Spain.

3. Servicio de Cardiología, Instituto de Investigación Biomédica A Coruña (INIBIC), Complejo Hospitalario Universitario A Coruña, CIBERCV Enfermedades Cardiovasculares, A Coruña. Spain.

4. Servicio de Cardiología, Instituto de Investigación Biomédica de Salamanca (IBISAL), Hospital Universitario de Salamanca, Universidad de Salamanca (USAL), CIBERCV Enfermedades Cardiovasculares, Salamanca, Spain.

*These authors contributed equally to this work: Luis M. Pérez-Belmonte and Inmaculada Moreno-Santos.

$\square$ Corresponding author: Luis M. Pérez-Belmonte MD, PhD. Address: Unidad de Gestión Clínica del Corazón, Hospital Clínico Universitario Virgen de la Victoria. Campus Universitario de Teatinos, s/n. Málaga, Spain. Phone: 0034951032672. E-mail: luismiguelpb@uma.es. Manuel Jiménez-Navarro. Address: Unidad de Gestión Clínica del Corazón, Hospital Clínico Universitario Virgen de la Victoria. Campus Universitario de Teatinos, s/n. Málaga, Spain. Phone: 0034951032672. E-mail: jimeneznavarro@secardiología.es.

(1) Ivyspring International Publisher. This is an open access article distributed under the terms of the Creative Commons Attribution (CC BY-NC) license (https://creativecommons.org/licenses/by-nc/4.0/). See http://ivyspring.com/terms for full terms and conditions.

Received: 2017.02.28; Accepted: 2017.04.25; Published: 2017.07.20

\begin{abstract}
Epicardial adipose tissue has been proposed to participate in the pathogenesis of heart failure. The aim of our study was to assess the expression of thermogenic genes (Uncoupling protein 1 (UCPI), peroxisome proliferator-activated receptor gamma coactivator 1-alpha (PGCl $\alpha$ ), and PR-domain-missing 16 (PRDM16) in epicardial adipose tissue in patients with heart failure, stablishing the difference according to left ventricular ejection fraction (reduced or preserved). Among the 75 patients in our study, 42.7\% $(n=32)$ had reduced left ventricular ejection fraction. UCPI, PGCl $\alpha$ and PRDM1 6 mRNA in EAT were significantly lower in patients with reduced left ventricular ejection fraction. Multiple regression analysis showed that age, male gender, body max index, presence of obesity, type-2-diabetes mellitus, hypertension and coronary artery disease and left ventricular ejection fraction were associated with the expression levels of UCPI, PGCl $\alpha$ and PRDM16 mRNA. Thermogenic genes expressions in epicardial adipose tissue (UCPI: OR 0.617, 95\%Cl 0.103-0.989, $\mathrm{p}=0.042$; PGCl $\alpha$ : OR 0.416, 95\%Cl 0.171-0.912, $\mathrm{p}=0.031$; PRDM16: OR $0.643,95 \% \mathrm{Cl} 0.116-0.997, \mathrm{p}=0.044)$ were showed as protective factors against the presence of heart failure with reduced left ventricular ejection fraction, and age (OR $1.643,95 \% \mathrm{Cl} 1.001-3.143$, $\mathrm{p}=0.026)$, presence of coronary artery disease $(\mathrm{OR} 6.743,95 \% \mathrm{Cl} 1.932-15.301, \mathrm{p}<0.001)$ and type-2-diabetes mellitus (OR 4.031, 95\% Cl 1.099-7.231, $\mathrm{p}<0.001)$ were associated as risk factors. The adequate expression of thermogenic genes has been shown as possible protective factors against heart failure with reduced ejection fraction, suggesting that a loss of functional epicardial adipose tissue brown-like features would participate in a deleterious manner on heart metabolism. Thermogenic genes could represent a future novel therapeutic target in heart failure.
\end{abstract}

Key words: Epicardial adipose tissue, heart failure, left ventricular ejection fraction, thermogenic genes. 


\section{Introduction}

Despite improvements in therapy, heart failure (HF) remains a leading cause of morbidity and mortality, affecting more than 37 million people worldwide and conferring a substantial burden on the health-care system [1]. It has been demonstrated that $\mathrm{HF}$ is associated with a pro-inflammatory state, mainly through an increase in pro-inflammatory adipokines and a decrease in anti-inflammatory adipokines, regulated by the expression of thermogenic genes [2]. Epicardial Adipose Tissue (EAT) has been proposed to participate in this adipokines production dysbalance and energy homeostasis, contributing to the pathogenesis of HF [3], but has not been fully characterized.

The main aim of our study was to assess the expression of thermogenic genes (Uncoupling protein 1 (UCP1), peroxisome proliferator-activated receptor gamma coactivator 1-alpha (PGC1a) and PR-domainmissing 16 (PRDM16) in EAT in patients with HF, stablishing the difference between patients with reduced ejection fraction (HFr-EF) and preserved ejection fraction (HFp-EF) and to evaluate the association with clinical and biochemical variables.

\section{Material and Methods}

\section{Patients}

Patients with HF who underwent elective cardiac surgery (coronary artery bypass and/or valve replacement) were included in our study and divided according to left ventricular ejection fraction (LVEF) determined by left ventriculography. HFr-EF was defined as an EF $\leq 40 \%$, whereas HFp-EF was defined as an $\mathrm{EF}>40 \%$. Exclusion criteria were severe infections, acute inflammatory diseases and/or cancer. Data about demographics and clinical characteristics, and biochemical parameters were collected.

The study was approved by the Institutional Research Ethics Committee from Hospital Universitario Virgen de la Victoria (Málaga, Spain) and carried out in accordance with the Declaration of Helsinki. Only patients who had previously given written informed consent were enrolled in this study.

\section{Biological material}

EAT biopsy samples (0.2-0.5g) were obtained near the proximal right coronary artery 1 hour after anesthesia. All the tissues were immediately frozen in liquid nitrogen and stored at $-80^{\circ} \mathrm{C}$ for RNA isolation.

In addition, peripheral venous blood was obtained and drawn into pyrogen-free tubes with or without ethylenedianminetetraacetic acid (anticoagulant). For serum, the tubes were left at room temperature for $20 \mathrm{~min}$ and then centrifuged at $1500 \mathrm{~g}$ for $10 \mathrm{~min}$ at $4^{\circ} \mathrm{C}$. In the hospital laboratory, fasting glucose, glycated hemoglobin (HbA1c), total cholesterol, low-density lipoprotein (LDL), high-density lipoprotein (HDL), triglycerides, creatinine, uric acid, glutamic-oxolacetic transaminase (GOT), glutamate-piruvate transaminase (GPT), gamma-glutamyl transferase (GGT), C-reactive protein (CRP), calcium, sodium and potassium were measured in a Dimension autoanalyzer (Dade Behring Inc., Deerfield, IL) by enzymatic methods (Randox Laboratories, Ldt., UK).

The gene expression levels in the adipose tissue were determined by real time quantitative polymerase chain reaction (PCR) using a predesigned and validated Taqman primer/probe sets.

\section{Statistical analysis}

Continuous variables are summarized as mean \pm standard deviation with Student's $\mathrm{T}$ test used to test the significance of between-group differences. Discrete variables are presented as frequencies and percentages with between-group differences tested using Pearson chi-square test. Multiple regression analysis were used in order to identify independent predictors of EAT UCP1, PGC1a and PRDM16 levels, as well as to control for confounding factors; and those clinical variables that achieved $\mathrm{P}<0.05$ on between-group comparison and cardiovascular plausible variables were included in the model. Logistic regression analysis was used to define the risk factors of reduced LVEF, and odds ratio (OR) and 95\% Confidence Interval $(95 \% \mathrm{CI})$ were calculated. SPSS for Windows version 15 (SPSS Inc. Chicago, IL, USA) was used for analyses and values were considered significant at $\mathrm{P}<0.05$.

\section{Results}

Among the 75 patients in our study, 42.7\% $(n=32)$ had reduced LVEF. Clinical and laboratory differences between patients with reduced and preserved LVEF HF are listed in Table 1. Among patients with reduced LVEF, there were more men and more likely to have coronary artery disease and obesity, and less valve heart disease.

UCP1, PGC1 $a$ and PRDM16 mRNA in EAT were significantly lower in patients with reduced LVEF ( $\mathrm{P}=0.004, \mathrm{P}=0.002$ and $\mathrm{P}=0.02$, respectively) (Figure 1 ).

Multiple regression analysis showed that age, male gender, body max index (BMI), presence of obesity, type-2-diabetes mellitus (DM2), hypertension and coronary artery disease and LVEF were 
independently associated with EAT UCP1, PGC1a, and PRDM16 mRNA levels (Table 2).

Thermogenic genes expressions in EAT were showed as protective factors against the presence of
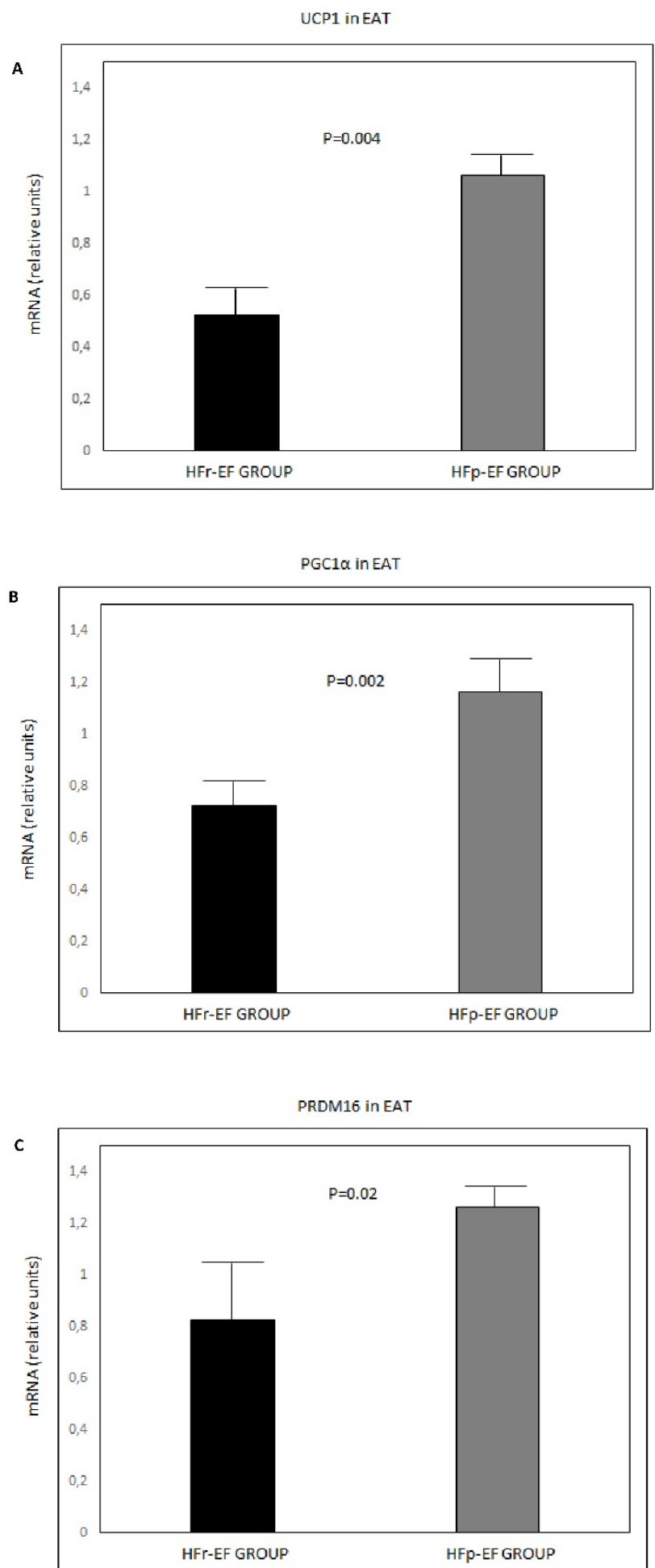

Figure 1. UCP1 (A), PGCl $\alpha(B)$ and PRDM16 (C) mRNA expression in EAT comparison between groups. Values are shown as mean \pm standard deviation. Values were considered to be statistically significant when $\mathrm{P}<0.05$. EAT: epicardial adipose tissue; HFp-EF: heart failure preserved-ejection fraction; HFr-EF: heart failure reduced ejection fraction; PGCla: peroxisome proliferator-activated receptor gamma coactivator 1-alpha; PRDM16: PR-domain-missing 16; UCP1: uncoupling protein 1
HFr-EF, and age, presence of coronary artery disease and type-2-diabetes mellitus were associated as risk factor in the logistic regression analysis (Table 3 ).

Table 1. Clinical and laboratory characteristics of patients with heart failure with reduced and preserved left ventricular ejection fraction

\begin{tabular}{|c|c|c|c|}
\hline $\begin{array}{l}\text { Variables } \\
\mathrm{N}(\%)\end{array}$ & $\begin{array}{l}\text { HFr-EF } \\
(n=32)\end{array}$ & $\begin{array}{l}\text { HFp-EF } \\
(n=43)\end{array}$ & $P$ value \\
\hline Age, years & $62.5 \pm 10.3$ & $62.8 \pm 11.5$ & 0.718 \\
\hline Male gender & $26(81.3)$ & $28(65.1)$ & 0.003 \\
\hline Body mass index, $\mathrm{kg} / \mathrm{m}^{2}$ & $26.6 \pm 4.4$ & $29.4 \pm 5.3$ & 0.03 \\
\hline LVEF, \% & $34.9 \pm 3.9$ & $60.4 \pm 8.5$ & $<0.001$ \\
\hline \multicolumn{4}{|l|}{ Cardiovascular risk factors } \\
\hline Current smoking & $14(43.8)$ & $16(37.2)$ & 0.267 \\
\hline Dyslipidemia & $15(46.9)$ & $23(53.5)$ & 0.317 \\
\hline Hypertension & $17(53.1)$ & $25(58.1)$ & 0.296 \\
\hline Diabetes mellitus & $10(31.3)$ & $16(37.2)$ & 0.277 \\
\hline Obesity & $14(43.8)$ & $21(48.8)$ & 0.127 \\
\hline Coronary artery disease & $19(59.4)$ & $17(39.5)$ & 0.04 \\
\hline Multivessel coronary disease & $22(68.8)$ & $30(69.8)$ & 0.431 \\
\hline Valve heart disease & $15(46.9)$ & $28(65.1)$ & 0.03 \\
\hline Cerebrovascular disease & $3(9.4)$ & $3(7)$ & 0.442 \\
\hline \multicolumn{4}{|l|}{ Medications } \\
\hline Aspirin & $17(53.1)$ & $24(55.8)$ & 0.766 \\
\hline Statin & $14(43.8)$ & $22(51.2)$ & 0.104 \\
\hline ACEI/ARB & $19(59.4)$ & $25(58.1)$ & 0.425 \\
\hline Beta-blocker & $21(65.6)$ & $31(72.1)$ & 0.370 \\
\hline \multicolumn{4}{|l|}{ Biochemical data } \\
\hline Glucose, mg/dL & $129.8 \pm 57.7$ & $122.1 \pm 43.7$ & 0.349 \\
\hline $\mathrm{HbA} 1 \mathrm{c}, \%$ & $6.6 \pm 1.3$ & $6.2 \pm 1.3$ & 0.721 \\
\hline Total cholesterol, mg/dL & $160 \pm 36$ & $163 \pm 42$ & 0.395 \\
\hline LDL cholesterol, mg/dL & $97 \pm 39$ & $98 \pm 33$ & 0.381 \\
\hline HDL cholesterol, mg/dL & $40 \pm 8.5$ & $39 \pm 14$ & 0.320 \\
\hline Triglycerides, mg/dL & $161 \pm 53$ & $144 \pm 61$ & 0.197 \\
\hline Creatinine, mg/dL & $1.3 \pm 0.8$ & $1 \pm 0.4$ & 0.711 \\
\hline Uric acid, mg/dL & $6.7 \pm 3.6$ & $5.6 \pm 1.9$ & 0.07 \\
\hline GOT, IU/L & $28.9 \pm 11.9$ & $35.3 \pm 37$ & 0.112 \\
\hline GPT, IU/L & $33.9 \pm 18.9$ & $36.9 \pm 29.1$ & 0.426 \\
\hline GGT, IU/L & $61.6 \pm 44.8$ & $52.1 \pm 57.6$ & 0.479 \\
\hline $\mathrm{CRP}, \mathrm{mg} / \mathrm{dL}$ & $27.1 \pm 46.6$ & $17 \pm 32.9$ & 0.222 \\
\hline Calcium, mg/dL & $8.5 \pm 0.7$ & $8.5 \pm 0.8$ & 0.858 \\
\hline Potassium, mmol/L & $4.2 \pm 0.7$ & $4.3 \pm 0.4$ & 0.855 \\
\hline Sodium, mmol/L & $136 \pm 4.4$ & $138 \pm 3.5$ & 0.342 \\
\hline
\end{tabular}

Values are shown as mean \pm standard deviation and frequencies (percentages). Values were considered to be statistically significant when $P<0.05$.

ACEI: Angiotensin Converting Enzyme Inhibitor; ARB: Antiotensin II Receptro Blocker; CRP: C-Reactive Protein; GGT: Gamma-Glutamyl Transferase; GOT: Glutamic-Oxolacetic Transaminase; GPT: Glutamate-Piruvate Transaminase; Hblac: glycated hemoglobin; HDL: High-Density Lipoprotein; HFp-EF: heart failure with preserved ejection fraction; HFr-EF: heart failure with reduce ejection fraction; IU/L: international units/liter; $\mathrm{kg} / \mathrm{m}^{2}$ : kilogram/square metre; LDL: Low-Density Lipoprotein; LVEF: left ventricular ejection fraction; $\mathrm{mg} / \mathrm{dL}$ milligram/deciliter;mmol/L: milimol/liter

\section{Discussion}

The present study found that patients with HFr-EF expressed significantly lower thermogenic genes (UCP1, PGC1a and PRDM16) in EAT than those with HFpEF. Age, male gender and different cardiovascular diseases were associated with the levels of thermogenic genes expression. EAT UCP1, PGC1a and PRDM16 mRNA levels were shown as possible protective factors against $\mathrm{HFr}-\mathrm{EF}$, and age and presence of CAD and DM2 were shown as risk factors. 
Table 2. Multiple regression analysis for prediction of epicardial adipose tissue UCPI, PGCl $\alpha$ and PRDM16 mRNA levels

\begin{tabular}{|c|c|c|c|c|c|c|c|c|c|}
\hline \multirow[t]{2}{*}{ Variables } & \multicolumn{3}{|c|}{ EAT UCP1 mRNA (R²=0.503) } & \multicolumn{3}{|c|}{ EAT PGC1a mRNA ( $\left.\mathrm{R}^{2}=0.641\right)$} & \multicolumn{3}{|c|}{ EAT PRDM16 mRNA (R²=0.499) } \\
\hline & $\beta$ & $95 \% \mathrm{CI}$ & P value & $\beta$ & $95 \% \mathrm{CI}$ & P value & $\beta$ & $95 \% \mathrm{CI}$ & $\mathrm{P}$ value \\
\hline Age & 0.071 & $0.019-0.132$ & 0.032 & 0.099 & $0.032-0.199$ & 0.003 & 0.079 & $0.041-0.177$ & 0.041 \\
\hline Gender (Man) & 0.119 & $-0.043-(-0.291)$ & 0.040 & -0.152 & $-0.064-(-0.237)$ & 0.001 & -0.101 & $-0.041-(-0.301)$ & 0.041 \\
\hline Body mass index & -0.090 & $-0.002-(-0.301)$ & 0.041 & -0.181 & $-0.001-(-0.248)$ & 0.039 & -0.088 & $-0.012-(-0.431)$ & 0.049 \\
\hline Obesity & -0.281 & $-0.108-(-0.931)$ & 0.029 & -0.381 & $-0.119-(-0.849)$ & 0.022 & -0.229 & $-0.099-(-0.983)$ & 0.041 \\
\hline Diabetes Mellitus & -0.230 & $-0.101-(-0.931)$ & 0.041 & -0.460 & $-0.159-(-0.869)$ & 0.044 & -0.201 & $-0.032-(-0.899)$ & 0.044 \\
\hline Hypertension & 0.083 & $0.021-0.333$ & 0.044 & 0.131 & $0.021-0.343$ & 0.039 & 0.072 & $0.012-0.435$ & 0.049 \\
\hline Dyslipidemia & 0.145 & $-0.241-2.001$ & 0.519 & 0.243 & $-0.343-1.141$ & 0.439 & 0.198 & $-0.341-1.191$ & 0.321 \\
\hline Coronary artery Disease & -0.111 & $-0.003-(-0.801)$ & 0.041 & -0.098 & $-0.003-(-0.798)$ & 0.038 & -0.131 & $-0.003-(-0.813)$ & 0.044 \\
\hline LVEF & 0.222 & $0.081-0.344$ & 0.002 & 0.399 & $0.049-0.598$ & 0.001 & 0.119 & $0.052-0.301$ & 0.002 \\
\hline
\end{tabular}

Values were considered to be statistically significant when $\mathrm{P}<0.05$

CI: Confidence Interval; EAT: epicardial adipose tissue; LVEF: left ventricular ejection fraction; PGC1a: peroxisome proliferator-activated receptor gamma coactivator 1-alpha; PRDM16: PR-domain-missing 16; UCP1: uncoupling protein 1.

Table 3. Logistic regression analysis for the presence of heart failure with reduced ejection fraction

\begin{tabular}{lll}
\hline Variable & OR $(95 \%$ CI $)$ & P value \\
\hline UCP1 mRNA & $0.617(0.103-0.989)$ & 0.042 \\
PGC1a mRNA & $0.416(0.171-0.912)$ & 0.031 \\
PRMD16 mRNA & $0.643(0.116-0.997)$ & 0.044 \\
Age & $1.643(1.001-3.143)$ & 0.026 \\
Gender (man) & $7.867(0.717-26.101)$ & 0.223 \\
Body mass index & $2.341(0.683-8.033)$ & 0.312 \\
Obesity & $3.001(0.843-12.301)$ & 0.323 \\
Diabetes mellitus & $4.031(1.099-7.231)$ & $<0.001$ \\
Hypertension & $2.499(0.798-14.133)$ & 0.492 \\
Dyslipidemia & $3.301(0.639-9.103)$ & 0.329 \\
Coronary artery disease & $6.743(1.932-15.301)$ & $<0.001$ \\
Values were considered to be statistically significant when P<0.05. \\
CI: confidence Interval; OR: odds ratio; PGC1a: peroxisome proliferator-activated \\
receptor gamma coactivator 1-alpha; PRDM16: PR-domain-missing 16; UCP1: \\
uncoupling protein 1.
\end{tabular}

These findings are important because they support the hypothesis that EAT thermogenic function could play an important role in the pathogenesis of HF. This is one of very few studies that have explored the influence of EAT on heart function in patients with $\mathrm{HF}$ and this is unique in that it assessed the association between thermogenic genes expression and HFr-EF and HFp-EF.

EAT represents a visceral brown-like adipose tissue located between the myocardium and the inner layer of visceral pericardium with a close anatomical proximity to the myocardium [4]. A functional EAT has been proposed to play a protector role over the myocardium but in pathological situations may be implicated in the development and/or progression of heart disease [3-5]. Several studies have shown that EAT is associated with the pathogenesis of HF, but focusing on EAT volume determined by echocardiography, magnetic resonance or computed tomography. Increased EAT thickness has been related to the severity of $\mathrm{HF}$ and explored the influence on diastolic and systolic functions [6,7]. However, only limited studies have explored the functionality of EAT [2,8]. Recent investigations have assessed the relationship between EAT gene expression in patients with HF, finding a functional role of EAT in the regulation of the development of HF [8]. p53, a tumor suppressor that coordinates DNA repair, cell cycle arrest and apoptosis; and adiponectin, an important anti-inflammatory adipokine, have been the principal gene expressions suggested to be important mediators of $\mathrm{HF}$ progression [9].

A number of reports have investigated the association between thermogenic gene expression such as UCP1, PGC1a and PRDM16, and coronary artery disease and other cardiovascular risk factors [10]. These genes have been recognized as specific marker of brown adipocites and regulators of oxidative metabolism and mitochondrial biogenesis, playing a relevant role in cardiac status [2]. A decrease of their gene mRNA expressions in EAT in patients with HFr-EF suggests a loss of EAT brown-like features, promoting pro-inflammatory and atherosclerotic pathways, exposing the heart to an excessive toxicity [11]. In line with these finding, we showed the thermogenic function of EAT and its involvement in the LVEF.

We acknowledge the following limitations in this study. We recruited a small number of recruited patients; our data were from a single hospital; and only small EAT biopsy samples were taken, being insufficient for a proteins determination. However, our study was carried out using a well-designed protocol and well-stablished methods. The hypothesis that EAT thermogenic genes expression was involved in patients with HF and influenced according to LVEF would need to be confirmed in a larger and multicenter research study.

In conclusion, the expression of thermogenic genes (UCP1, PGC1a and PRDM16) was lower in patients with HFr-EF than in those with HFp-EF. These genes have been shown as possible protective factors against HFr-EF, suggesting a loss of functional EAT brown-like features, what, subsequently, would participate in a deleterious manner on heart 
metabolism. Thermogenic genes could represent a future novel therapeutic target in patients with HFr-EF.

\section{Acknowledgements}

The authors thank the cardiac surgeons from Department of Heart Surgery (Virgen de la Vitoria Hospital, Málaga) for their contribution in collecting samples.

\section{Competing Interests}

This work was supported by grants from the Spanish Ministry of Health (FIS) (PI13/02542, PI11/01661) and Spanish Cardiovascular Research Network (RD12/0042/0030)/CIBERCV Enfermedades Cardiovasculares (CB16/11/00360) co-founded by Fondo Europeo de Desarrollo Regional (FEDER). Luis M. Pérez-Belmonte is supported from Red de Investigación Cardiovascular (RD12/0042/0030)/ CIBERCV Enfermedades Cardiovasculares (CB16/ 11/00360) (Contrato Post-MIR “Jordi Soler"), and Lourdes Garrido-Sáchez is supported by a fellowship from the Fondo de Investigación Sanitaria (FIS) "Miguel Servet I" (MS13/00188-CP13/00188). The authors have declared that no competing interests exist.

\section{References}

1. Mozaffarian, D, Benjamin EF, Go AS, et al. American Heart Association Statistics Committee; Stroke Statistics Subcommittee. Heart disease and stroke statistics-2016 Update: A Report From the American Heart Association. Circulation. 2016;133:38-360

2. Sacks HS, Fain JN, Holman B, et al. Uncoupling protein-1 and related messenger ribonucleic acids in human epicardial and other adipose tissues: epicardial fat functioning as brown fat. J Clin Endocrinol Metab. 2009;94:3611-5

3. Iacobellis G, Bianco AC. Epicardial adipose tissue: emerging physiological, pathophysiological and clinical features. Trends Endocrinol Metab. 2011;22:450-7.

4. Iacobellis G, Corradi D, Sharma AM. Epicardial adipose tissue: anatomic, biomolecular and clinical relationships with the heart. Nat Clin Pract Cardiovasc Med. 2005;2:536-43.

5. Pérez-Belmonte LM, Moreno-Santos I, Cabrera-Bueno F, et al. Expression of Sterol Regulatory Element-Binding Proteins in epicardial adipose tissue in patients with coronary artery disease and diabetes mellitus: preliminary study. Int J Med Sci. 2017;14:268-74.

6. Parisi V, Rengo G, Perrone-Filardi P, et al. Increased Epicardial Adipose Tissue Volume Correlates With Cardiac Sympathetic Denervation in Patients With Heart Failure. Circ Res. 2016;118:1244-53.

7. Tabakci MM, Durmuş HI, Avci A, et al. Relation of epicardial fat thickness to the severity of heart failure in patients with nonischemic dilated cardiomyopathy. Echocardiography. 2015;32:740-8.

8. Agra RM, Teijeira-Fernández E, Pascual-Figal D, et al. Adiponectin and p53 mRNA in epicardial and subcutaneous fat from heart failure patients. Eur J Clin Invest. 2014;44:29-37.

9. Iacobellis G, Bianco AC. Epicardial adipose tissue: emerging physiological, pathophysiological and clinical features. Trends Endocrinol Metab. 2011;22:450-7.

10. Moreno-Santos I, Pérez-Belmonte LM, Macías-González M, et al. Type 2 diabetes is associated with decreased PGC1a expression in epicardial adipose tissue of patients with coronary artery disease. J Transl Med. 2016;14:243.

11. Bartelt A, Bruns OT, Reimer R, et al. Brown adipose tissue activity controls triglyceride clearance. Nat Med. 2011;17:200-5. 